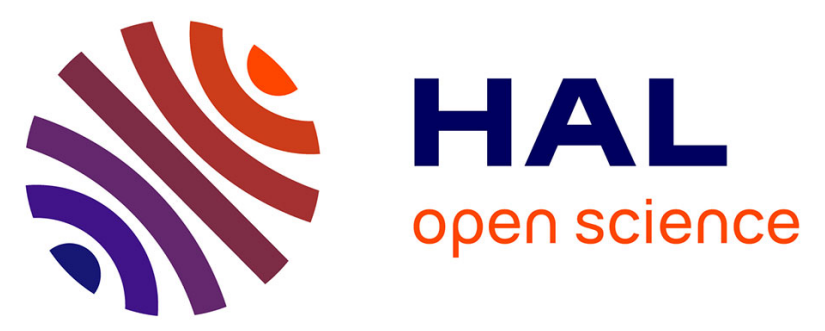

\title{
Self-Guided Propagation of Ultrashort Laser Pulses in the Anomalous Dispersion Region of Transparent Solids: A New Regime of Filamentation
}

Magali Durand, Amélie Jarnac, Aurélien Houard, Yi Liu, Stéphanie Grabielle, Nicolas Forget, Anne Durécu, Arnaud Couairon, André Mysyrowicz

\section{To cite this version:}

Magali Durand, Amélie Jarnac, Aurélien Houard, Yi Liu, Stéphanie Grabielle, et al.. Self-Guided Propagation of Ultrashort Laser Pulses in the Anomalous Dispersion Region of Transparent Solids: A New Regime of Filamentation. Physical Review Letters, 2013, 110 (11), pp.115003. 10.1103/PhysRevLett.110.115003 . hal-00852051

\section{HAL Id: hal-00852051}

https://hal-polytechnique.archives-ouvertes.fr/hal-00852051

Submitted on 19 Aug 2013

HAL is a multi-disciplinary open access archive for the deposit and dissemination of scientific research documents, whether they are published or not. The documents may come from teaching and research institutions in France or abroad, or from public or private research centers.
L'archive ouverte pluridisciplinaire HAL, est destinée au dépôt et à la diffusion de documents scientifiques de niveau recherche, publiés ou non, émanant des établissements d'enseignement et de recherche français ou étrangers, des laboratoires publics ou privés. 


\title{
Self guided propagation of ultrashort laser pulses in the anomalous dispersion region of transparent solids: a new regime of filamentation
}

\author{
M. Durand ${ }^{1 *}$, A. Jarnac ${ }^{1}$, A. Houard ${ }^{1}$, Yi Liu ${ }^{1}$, S. Grabielle ${ }^{2}$, N. Forget ${ }^{2}$, A. Durécu ${ }^{3}$, A. Couairon ${ }^{4}$ \\ and A. Mysyrowicz ${ }^{1, * *}$ \\ ${ }^{1}$ Laboratoire d'Optique Appliquée, ENSTA ParisTech, Ecole Polytechnique, CNRS, F-91761 Palaiseau, France \\ ${ }^{2}$ FASTLITE, Centre Scientifique d'Orsay, Plateau du Moulon, F-91401 Orsay, France \\ ${ }^{3}$ Onera - The French Aerospace Lab, BP 80100, F-91123 Palaiseau cedex, France \\ ${ }^{4}$ Centre de Physique Théorique, CNRS, Ecole Polytechnique, F-91128 Palaiseau, France \\ * now at CREOL, University of Central Florida, Orlando, FL 32816, USA \\ **andre.mysyrowicz@ensta.fr
}

\begin{abstract}
We report measurements concerning the propagation of femtosecond laser pulses in fused silica with a wavelength at $1.9 \mu \mathrm{m}$ falling in the negative GVD region. Under sub-GW excitation power, stable filaments are observed over several $\mathrm{cm}$ showing the emergence of non-spreading pulses both in space and time. At higher excitation powers, one observes first multiple pulse splitting followed by the emergence of the quasi spatio-temporal solitary filament. These results are well reproduced by numerical simulations.
\end{abstract}

The propagation of an intense ultrashort laser pulse in a transparent medium, despite the apparent simplicity of the problem, is a complex process. The nonlinear propagation is first dominated by the optical Kerr response of the medium. The light-induced change of refractive index leads to beam selffocusing [1], pulse spectral broadening [2] and beam profile reshaping [3, 4]. For a pulse peak power above a critical value $P_{c r}=3.77 \lambda_{0}^{2} / 8 \pi n_{0} n_{2}$, where $\lambda_{0}$ is the laser wavelength in vacuum, $n_{0}$ the corresponding refractive index and $n_{2}$ the nonlinear Kerr index, diffraction is unable to arrest the beam self focusing effect. The intensity grows to the point that strong field ionization of air molecules occurs. This prevents beam collapse because the generated plasma consumes laser energy and induces a defocusing of the beam. The further evolution of the pulse is ruled by a competition between diffraction, group velocity dispersion, the increase of the refractive index from the optical Kerr effect, multiphoton absorption and the reduction of refractive index due to plasma formation. The combination of these effects leads to filamentation, a process where the pulse maintains a narrow diameter and high peak intensity over impressive distances which can reach several $\mathrm{km}$ in atmosphere $[5,6]$. Most experiments on filamentation so far have concentrated on situations where the laser wavelength corresponds to a region of normal group velocity dispersion (GVD). Filamentary behavior has been observed as a generic trend in transparent gases, solids and liquids. However this universal trend masks a complex underlying situation. The filament length and plasma density depend critically on the initial pulse intensity, initial pulse duration and beam convergence [7]. Plasma and GVD induced pulse splitting lead to pulse self shortening with a single compressed pulse component emerging fortuitously at some distance, followed by a rapid deterioration [8]. As a consequence, it is hard to achieve a controlled and stable filamentation regime with preserved laser pulse features over extended distances.

A simpler behavior is expected when the laser wavelength falls in the anomalous dispersion region. New red (blue) frequencies generated by self phase modulation on the ascending (descending) slopes of the pulse are now swept back to the peak of the pulse, instead of being dispersed as in the normal case. As a consequence, a simultaneous time and space compression occurs which favors a new type of filamentation where the self-guided pulses do not spread in time and space over long distances [9]. Several authors have started to investigate this regime, both experimentally and theoretically. On the theoretical front, Shim et al. [10] have simulated filamentation in air at $3.1 \mu \mathrm{m}$, a region of negative GVD. The authors conclude that this medium is unfavorable for a clear demonstration of temporal solitons because pulse self compression is restricted by a limited range of negative dispersion (approximately $200 \mathrm{~nm}$ around $3.1 \mu \mathrm{m}$ ). The situation is more favorable in condensed media such as fused silica $\left(\mathrm{SiO}_{2}\right)$, where a broad spectral bandwidth of negative GVD extending over more than 2000 $\mathrm{nm}$ beyond $1.26 \mu \mathrm{m}$ is available. Several authors have performed numerical simulations of the 
propagation of IR laser pulses in fused silica [11-13]. On the experimental side, measurements in fused silica have shown that the critical threshold for filamentation is reduced [12] and that a broad conical continuum emission is produced [14, 15], suggesting efficient pulse self compression. However, to the best of our knowledge, there is no report yet on the free propagation of a stable, self compressed pulse over long distances.

In this letter we report measurements concerning the propagation of femtosecond laser pulses in fused silica with a central wavelength falling in the negative GVD region. Impressive stability of self-guided pulses is observed in the time and space domain, showing the emergence of a quasi spatio-temporal soliton. Our results are well reproduced by numerical simulations.

Infrared laser pulses at $1.9 \mu \mathrm{m}$ of $40 \mathrm{fs}$ duration and energy between 8 and $20 \mu \mathrm{J}$ were weakly focused close to the front surface of a $3 \mathrm{~cm}$ thick block of fused silica (beam numerical aperture NA $=0.015$ ). Side images recorded with a CCD camera of the blue luminescence of $\mathrm{SiO}_{2}$ reveal a thin filamentary track extending over a large part of the sample (see Fig. 1). The blue luminescence induced by multiphoton absorption of the propagating laser pulse becomes apparent at laser pulse intensities I > $10^{12} \mathrm{~W} / \mathrm{cm}^{2}$. In Figure 1, we compare the filaments obtained for a similar ratio $P / P_{c r} \sim 15$ for a laser pump wavelength at $0.8 \mu \mathrm{m}$ (normal dispersion, $P_{c r}=3 \mathrm{MW}$, Figure 1(a)) and $1.9 \mu \mathrm{m}$ (anomalous dispersion region, $P_{c r}=16 \mathrm{MW}$, Figure 1(b)). Propagation with positive GVD generates a significantly shorter filament with multiple refocusing cycles. Figure $1 \mathrm{c}$ also shows the filament at $1.9 \mu \mathrm{m}$ obtained at higher input powers $P>25 P_{c r}$. In this case, multiple focusing defocusing cycles appear first, followed by a continuous and uniform filament similar to that seen in Figure 1(b).

We have measured the diameter of the infrared filament obtained with light at $1.9 \mu \mathrm{m}$ with $P \sim 18 P_{c r}$. The spatial profile of the laser pulse emerging from the back surface of the sample was imaged by a silica lens with a focal distance of $10 \mathrm{~cm}$ onto a camera. The filament length at which the diameter was measured was varied by displacing the focus of the converging beam towards the interior of the sample, effectively bringing the filament closer to the sample rear surface. A circular beam with a constant diameter (FWHM) of $20 \mu \mathrm{m}$ is obtained (Fig. 2(a)). By comparison, the beam profile recorded at an equivalent distance in the absence of sample shows a larger asymmetric pattern (see Fig. 2(b)). Spatial self compression and self cleaning of the laser beam profile is a telltale of filamentation [4].

A single-shot, self-referenced spectral interferometry technique called Wizzler [16] operating in the Near Infrared region, was chosen to characterize the duration of the self-guided pulse emerging from the sample. It is free from the coherence artifact due to shot-to-shot instability that can arise with multi-shot characterization techniques [17]. As the signal-to-noise ratio on the spectrometer was low, the measurements were integrated over ten shots. Pulse duration of $20 \mathrm{fs}$ was retrieved, corresponding to a self compression factor of 2 (see Fig. 3). Considering the small number of averaged shots and the reproducibility of the 20 fs pulse duration measurement, we can conclude that free propagation of an ultrashort laser pulse in the negative group velocity dispersion region of a transparent medium can lead to the formation of a stable self-guided pulse holding the attributes of a temporal solitary wave within a range of incident laser powers $P_{c r}<P<25 P_{c r}$. However, it is necessary to stress that this self guided pulses are intrinsically lossy and therefore cannot qualify stricto sensu as genuine solitons. Indeed energy is consumed by multiphoton ionization at every step of the pulse progression. Recent papers have described such stationary and lossy optical pulses by the terms of $O$-waves or dissipative light bullets acting as attractors for the nonlinear pulse propagation in a Kerr medium with anomalous dispersion $[18,19]$. We also note that at high excitation $P>30 P_{c r}$, the laser pulse experiences a spectral broadening with bluer components reaching the normal dispersion region. A detailed study of this broadening will be published elsewhere.

We have performed simulations of pulse propagation in the anomalous dispersion region of fused silica under conditions close to the experiments. The electric field is written as a complex envelope $E_{\omega_{0}}(\vec{r}, t, z)$ and a carrier at frequency $\omega_{0}$ :

$$
E=E_{\omega_{0}} \exp \left\lfloor i\left(k_{\omega_{0}} z-\omega_{0} t\right)\right\rfloor
$$


with $k_{\omega_{0}} \equiv k\left(\omega_{0}\right)$, where $k(\omega)$ denotes the dispersion relation in fused silica [20]. The propagation equation for the complex envelope $\hat{E}_{\omega_{0}}\left(k_{\perp}, \omega, z\right)$ in the Fourier domain takes the form of a canonical propagation equation [21]:

$$
\frac{\partial \hat{E}_{\omega_{0}}}{\partial z}=i K_{\omega_{0}}\left(\omega, k_{\perp}\right) \hat{E}_{\omega_{0}}+i Q_{\omega_{0}}(\omega)\left(\frac{\hat{P}_{\omega_{0}}}{\varepsilon_{0}}+\frac{i}{\omega} \frac{\hat{J}_{\omega_{0}}}{\varepsilon_{0}}\right)
$$

Here the first (linear) term on the right hand side represents diffraction, dispersion and space-time coupling: $\quad K_{\omega_{0}}\left(\omega, k_{\perp}\right) \equiv \frac{1}{2}\left(k_{\omega_{0}}+k_{\omega_{0}}^{\prime}\left(\omega-\omega_{0}\right)\right)^{-1}\left[k^{2}(\omega)-\left(k_{\omega_{0}}+k_{\omega_{0}}^{\prime}\left(\omega-\omega_{0}\right)\right)^{2}-k_{\perp}^{2}\right], \quad$ where the dispersive properties of the medium are described by a Sellmeier-like relation $k(\omega)$, and $k_{\omega_{0}} \equiv k\left(\omega_{0}\right)$, $k_{\omega_{0}}^{\prime} \equiv d k /\left.d \omega\right|_{\omega_{0}}$. The nonlinear dispersion function reads $Q_{\omega_{0}}(\omega) \equiv \frac{1}{2}\left(k_{\omega_{0}}+k_{\omega_{0}}^{\prime}\left(\omega-\omega_{0}\right)\right)^{-1}\left(\omega^{2} / c^{2}\right)$. These frequency dependent operators in equations (2) generalize the standard nonlinear Schrödinger equation so as to properly describe nonlinear propagation of pulses with broad spectra, as short as a single cycle. The derivation is explained in details in refs. [21]. In the temporal domain, pulse propagation is described in the moving frame $\tau=t-k_{0}^{\prime} z$, where $t$ denotes time in the laboratory frame. The nonlinear terms in Eq. (2) are all included in the nonlinear polarization envelope $\hat{P}_{\omega_{0}}\left(k_{\perp}, \omega, z\right)$ and current envelope $\hat{J}_{\omega_{0}}\left(k_{\perp}, \omega, z\right)$, acting as source terms for the propagation. The nonlinear polarization describes the optical Kerr effect:

$$
\frac{P_{\omega_{0}}}{\varepsilon_{0}}=2 n_{\omega_{0}} n_{2}\left|E_{\omega_{0}}\right|^{2} E_{\omega_{0}}
$$

The current describes plasma absorption and plasma defocusing in the framework of the Drude model, with collision time $\tau_{c}$ :

$$
\frac{J_{\omega_{0}}}{\varepsilon_{0}}=c \sigma_{\omega_{0}}\left(1+i \omega_{0} \tau_{c}\right) \rho E_{\omega_{0}}+c n_{\omega_{0}} \beta_{K}\left|E_{\omega_{0}}\right|^{2 K-2} E_{\omega_{0}}
$$

The various parameters in Eqs. (3-4) are: the linear refractive index $n_{\omega_{0}} \approx 1.43$, the cross section for inverse Bremsstrahlung $\sigma_{\omega_{0}}=4.7 \times 10^{-21} \mathrm{~cm}^{2}$ calculated from the Drude model with the collision time $\tau_{c}=3 \mathrm{fs}$ in fused silica, the cross section for multiphoton absorption $\beta_{K}=3.1 \times 10^{-164} \mathrm{~W}^{-13} \cdot \mathrm{cm}^{25}$, the number of photons $K=14$ involved in the process at $1900 \mathrm{~nm}$. Multiphoton absorption coefficients are estimated from the Keldysh model for dielectrics [22, 23]. The nonlinear index coefficient $n_{2}=2.3 \times 10^{-16} \mathrm{~cm}^{2} / \mathrm{W}$ is slightly lower than the value $n_{2}=2.45 \times 10^{-16} \mathrm{~cm}^{2} / \mathrm{W}$ measured at $804 \mathrm{~nm}$ [24] due to a small effect of dispersion from 800 to $1900 \mathrm{~nm}$. The role of the retarded Kerr effect was neglected as no significant Raman shift appeared in the measured spectra.

Ionization of fused silica is described by a single rate equation for the generation of free electrons with density $\rho$ including source terms corresponding to multiphoton ionization, avalanche and recombination processes:

$$
\frac{\partial \rho}{\partial t}=\sigma_{K}\left|E_{\omega_{0}}\right|^{2 K}\left(\rho_{n t}-\rho\right)+\sigma_{\omega_{0}}\left|E_{\omega_{0}}\right|^{2} \frac{\rho}{U_{i}}-\frac{\rho}{\tau_{r}}
$$

where $\sigma_{K}=1.0 \times 10^{-168} \mathrm{~cm}^{28} \cdot \mathrm{W}^{-14} \cdot \mathrm{s}^{-1}$ denotes the cross section for multiphoton ionization at $1900 \mathrm{~nm}$, $\rho_{n t} \approx 2.1 \times 10^{22} \mathrm{~cm}^{-3}$ denotes the density of neutral molecules, $U_{i}=9 \mathrm{eV}$ denotes the ionization potential of fused silica, and $\tau_{r}=150 \mathrm{fs}$ denotes a characteristic time for recombination [25].

For input powers close to $P_{c r}$, the numerical simulation reproduces well the observed features of Figure $1 \mathrm{~b}$. Simulations reveal the formation of a self-guided pulse with a fluence diameter of $20 \mu \mathrm{m}$ (FWHM) which starts after $0.5 \mathrm{~cm}$ of propagation and persists over the remaining sample length (Fig. 4(a)-(b) and Fig. 5(a)). The calculated pulse duration (FWHM) is 8 fs showing that the filament pulse 
reaches basically the single optical cycle limit (Fig. 4(c)). Our measurements show a longer duration because of the limited time resolution of the Wizzler system and because no provision was taken during the measurement to remove the laser energy reservoir surrounding the filament, of longer duration [26]. By performing a spatially integrated calculation over a larger beam diameter, one obtains a duration of $20 \mathrm{fs}$, in agreement with the measurement (see Fig. 4(c)). Simulations at incident laser power exceeding $10 P_{c r}$ show a more complex situation at the initial stage of filamentation. The pulse undergoes first rapid variations of size and duration before a stationary pulse is formed (see Fig. 1 (c) and Fig. 5 (b)).

We believe that quasi spatio-temporal solitons emerging from filamentation can be found in many other transparent media with a broad region of negative group velocity dispersion. We stress however that such quasi-solitons are formed within a range of input power filamentation close to the critical power. Filamentation in the anomalous dispersion region of transparent solids should provide a simple method to produce ultrashort laser pulses with clean beam profile and well defined intensity in the range of terawatt $/ \mathrm{cm}^{2}$. Such optical pulses can be useful in many applications where an intense ultrashort laser pulse of known characteristics is required.

\section{Acknowledgments}

We acknowledge technical help from M. Lozano, D. Boschetto and K. Plamann. A. Jarnac acknowledges partial support from French DGA.

\section{References}

[1] J. H. Marburger, Prog. Quant. Electr. 4, 35 (1975)

[2] The Supercontinuum Laser Source, edited by R. R. Alfano, Springer-Verlag, New York (1989)

[3] K. D. Moll, A. L. Gaeta, and G. Fiebich, Phys. Rev. Lett. 90, 203902 (2003)

[4] B. Prade, M. Franco, A. Mysyrowicz, A. Couairon, H. Buersing, B. Eberle, M. Krenz, D. Seiffer, O. Vasseur, Opt Lett. 31, 2601 (2006)

[5] A. Couairon, A. Mysyrowicz, Phys. Reports, 441, 47 (2007)

[6] J. Kasparian et al., Science 301, 61 (2003)

[7] Y.-H. Chen, S. Varma, T. M. Antonsen and H. M. Milchberg, Phys. Rev. Lett. 105, 215005 (2010)

[8] A. Couairon et al., J. Mod. Opt. 53, 75 (2006)

[9] F. Wise and P. Di Trapani, Optics and Photonics News February, 28 (2002)

[10] B. Shim, S. E. Schrauth and A.L. Gaeta, Opt. Express 19, 9118 (2011)

[11] J. Liu, R. Li and Z. Xu, Phys. Rev. A 74, 043801 (2006)

[12] K. D. Moll and A. L. Gaeta, Opt. Lett. 29, 995 (2004)

[13] L. Bergé and S. Skupin, Phys. Rev. E 71, 06560 (2005)

[14] A. Saliminia, S. L. Chin and R. Vallée, Opt. Express 13, 5731 (2005)

[15] D. Faccio et al., Phys. Rev. E 74, 047603 (2006)

[16] T. Oksenhendler, S. Coudreau, N. Forget, V. Crozatier, S. Grabielle, R. Herzog, D. Kaplan and O. Gobert, App. Phys. B 99, 7 (2010)

[17] J. Ratner, G. Steinmeyer, T. C. Wong, R. Bartels and R. Trebino, Opt. Lett. 37, 2874 (2012)

[18] M. A. Porras, A. Parola and P. Di Trapani, J. Opt. Soc. Am. B 22, 1406 (2005)

[19] M. A. Porras, Opt. Express 18, 7376 (2010)

[20] I. H. Malitson, J. Opt. Soc. Am. 55, 1205 (1965)

[21] A. Couairon, E. Brambilla, T. Corti, D. Majus, O. de J. Ramírez-Góngora and M. Kolesik, Eur. Phys. J. Special Topics 199, 5 (2011)

[22] L. V. Keldysh, Sov. Phys. JETP 20, 1307 (1965) [Zh. Eksp. Teor. Fiz. 47, 1945 (1964)]

[23] A. Couairon, L. Sudrie, M. Franco, B. Prade and A. Mysyrowicz, Phys. Rev. B 71, 125435

(2005)

[24] A. J. Taylor, G. Rodriguez and T. S. Clement, Opt. Lett. 21, 1812 (1996)

[25] P. Audebert et al., Phys. Rev. Lett. 73, 1990 (1994)

[26] A. Zaïr et al., Opt. Express 15, 5394 (2007) 


\section{FIGURES}

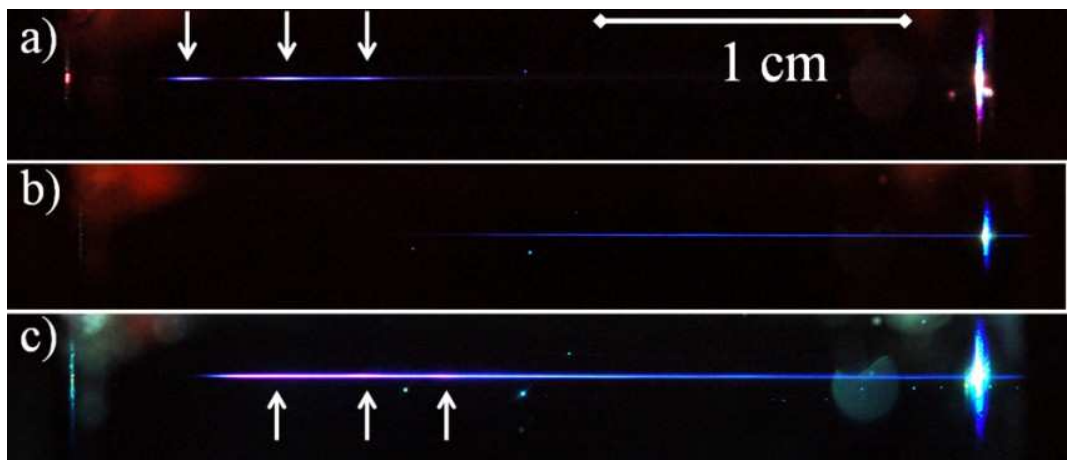

FIG. 1. Side view photography of the blue fluorescence of $\mathrm{SiO}_{2}$ induced by a self-guided laser pulse at a) $800 \mathrm{~nm}$ with $P=18 P_{c r}$, b) $1.9 \mu \mathrm{m}$ with $P=13 P_{c r}$ and c) $1.9 \mu \mathrm{m}$ with $P=30 P_{c r}$. The laser pulse is focused on a $70 \mu \mathrm{m}$ spot (FWHM) at the left surface of the $3 \mathrm{~cm}$ thick sample. Vertical arrows indicate the position of focusing-defocusing cycles.
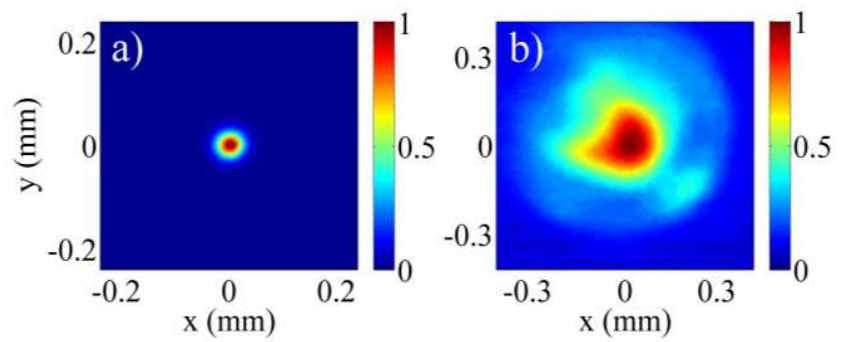

FIG. 2. Beam profile of the laser pulse at $1.9 \mu \mathrm{m}$ (a) with filamentation (b) without filamentation. Intensity scale is normalized.

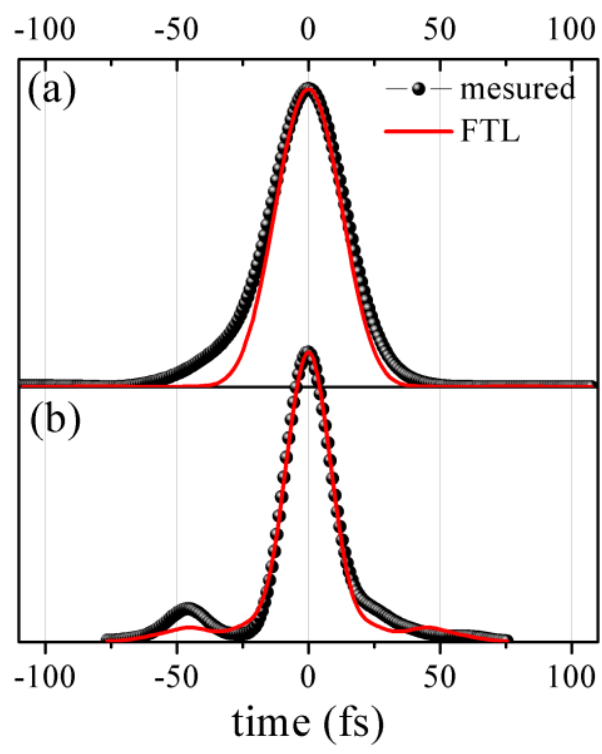

FIG. 3. Temporal pulse shape measured with the Wizzler for the initial pulse (40 fs FWHM) (a) and for filament lengths of $0.5 \mathrm{~cm}$ (20 fs FWHM) (b). Also shown in red is the Fourier Transform Limited pulse (FTL). 

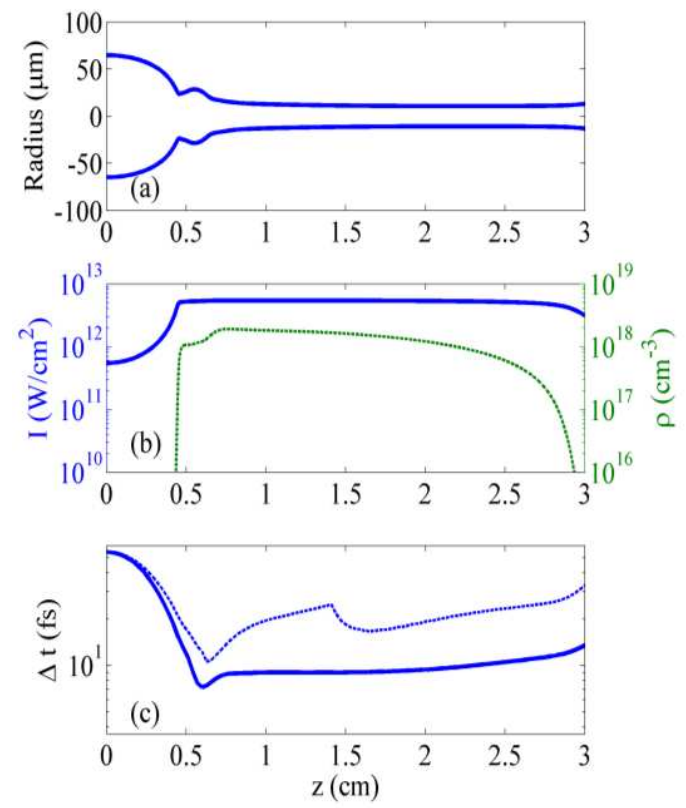

FIG. 4. Calculated results of the propagation through fused silica of an optical pulse at $1.9 \mu \mathrm{m}$ of 110 $\mu \mathrm{m}$ diameter $(\mathrm{FWHM})$ and $5 \times 10^{11} \mathrm{~W} / \mathrm{cm}^{2}$ intensity collimated on the front sample surface. a) Beam radius as a function of distance z. b) Intensity (full line) and free electron density (dotted green line) as a function of z. c) Pulse duration as a function of z. The blue line corresponds to the duration within the filament core of $25 \mu \mathrm{m}$ diameter, the red line corresponds to the duration obtained when averaging over a filament diameter of $50 \mu \mathrm{m}$.

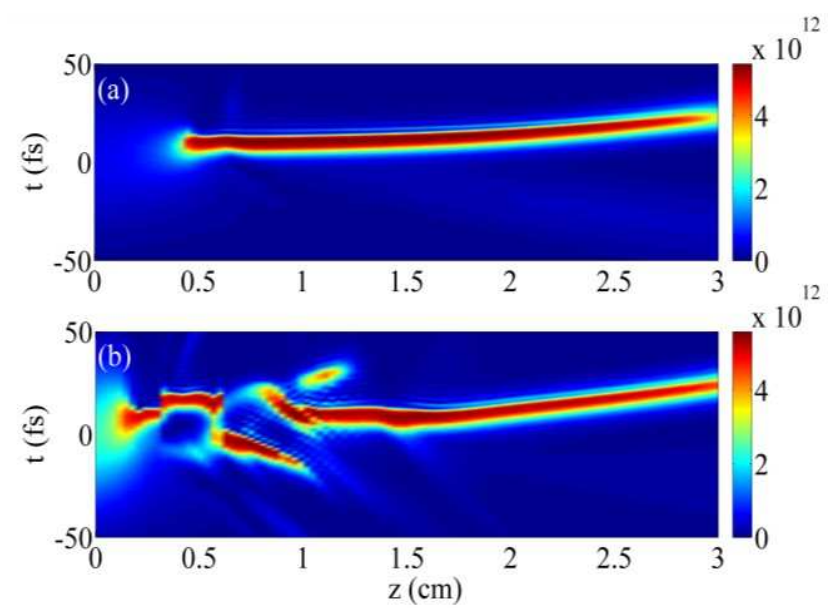

FIG. 5. Calculated time-space profile of the laser pulse for a laser pulse energy of $6 \mu \mathrm{J}$ (a) and $26 \mu \mathrm{J}$ (b) corresponding respectively to 7 and $30 P_{c r}$. 